\title{
Exploring Numeracy in Word Embeddings
}

\author{
Aakanksha Naik*, Abhilasha Ravichander*, \\ Carolyn Rose, Eduard Hovy \\ Language Technologies Institute, Carnegie Mellon University \\ \{anaik, aravicha, cprose, ehovy\}@cs.cmu.edu
}

\begin{abstract}
Word embeddings are now pervasive across NLP subfields as the de-facto method of forming text representataions. In this work, we show that existing embedding models are inadequate at constructing representations that capture salient aspects of mathematical meaning for numbers, which is important for language understanding. Numbers are ubiquitous and frequently appear in text. Inspired by cognitive studies on how humans perceive numbers, we develop an analysis framework to test how well word embeddings capture two essential properties of numbers: magnitude (e.g. $3<4$ ) and numeration (e.g. $3=$ three). Our experiments reveal that most models capture an approximate notion of magnitude, but are inadequate at capturing numeration. We hope that our observations provide a starting point for the development of methods which better capture numeracy in NLP systems.
\end{abstract}

\section{Introduction}

Word embeddings operationalize the distributional hypothesis, where a word is characterized by "the company it keeps" (Harris, 1954; Firth, 1957), and have been shown to capture semantic regularities in vector space (Mikolov et al., 2013c). They have been a driving force in NLP in recent years, and enjoy widespread use in a variety of semantic tasks (Rumelhart et al.; Mikolov et al., 2013a,b; Collobert and Weston, 2008; Glorot et al., 2011; Turney and Pantel, 2010; Turney, 2013).

However, to what extent do these word representations capture numeric properties? Numbers often need to be dealt with precisely, and understanding the meaning of text also requires a careful understanding of the quantities involved. They have been identified to play an important role in textual entailment, a benchmark natural language

\footnotetext{
*The first two authors contributed equally to this work.
}

understanding task. Marneffe et al. (2008) extract pairs of contradictions that occur naturally on Wikipedia and Google News, and find that as many as $29 \%$ of contradictions arise due to numeric discrepancies. They also identify that on many Recognizing Textual Entailment (RTE) datasets, $8.8 \%$ of contradictory pairs feature numeric contradictions. Naik et al. (2018) find that model inability to do numerical reasoning causes $4 \%$ of errors made by state-of-the-art models in Natural Language Inference. Spithourakis and Riedel (2018) emphasize the importance of numeracy in language modeling. Yet, numbers are often forgotten and even masked in NLP applications (Mitchell and Lapata, 2009).

In several domains such as economics, finance and scientific articles numbers can play a crucial role in text. Take for example a recent news headline,

Met Office: Global Warming could exceed
$1.5 \mathrm{C}$ within five years

Ideally, the text representation we use should be able to capture that global warming can exceed 1.5 C, not 100 C. Magnitude is an essential aspect of a number's meaning ${ }^{1}$ (Dehaene et al., 1998; Whalen et al., 1999; Cantlon and Brannon, 2006; Gross, 2011; Cutini and Bonato, 2012; Agrillo et al., 2012; Feigenson et al., 2004) . Systems should also be able to draw valid inferences irrespective of whether the text uses "five" or " 5 ". This requires an understanding of symbolic representations used to record numbers in text. Such representation systems are called numeration systems, and individual symbols within the system

\footnotetext{
${ }^{1}$ Prior work has shown that humans, as well as several species of animals share analogue systems that represent "quantities" or "magnitudes" associated with numbers(Dehaene et al., 1998)
} 
are called numerals $^{2}$. Systems must handle numeration, i.e. associations between distinct symbols used for the same number under different systems $(3=$ three).

In this work, we examine the extent to which word embeddings are capable of representing numeracy attributes, asking the question - if pretrained word embeddings are utilized for representing text across NLP tasks, what can they represent about numbers? Our framework formulates triples of numbers to probe word embeddings on their ability to represent magnitude, and their robustness to differences in numeration. We hope this analysis highlights limitations of current pretrained word embeddings at capturing numeracy, and will motivate future research to develop more careful treatments of quantities in text.

\section{Analysis Framework}

We construct an analysis framework to evaluate embeddings on their ability to capture magnitude and numeration. Numbers follow a well-defined ordering, under a mathematical system, which holds independent of textual context (e.g.: $0<$ $1<2 \ldots$ ). This ordering is established by magnitude (Izard and Dehaene, 2008; Russell, 2009) and is consistent across numeration systems. Therefore, an embedding representation that captures magnitude and numeration precisely should maintain this ordering across numeration systems in the embedding space. We evaluate this ability by constructing contrastive tests (Zhu et al., 2018).

A contrastive test for a property $p$ is defined as a triple $\left(x, x_{+}, x_{-}\right)$such that $x$ is closer to $x_{+}$than $x_{-}$under $p$. If embeddings capture $p, x$ will be closer to $x_{+}$than $x_{-}$in the embedding space, indicating that the embedding method passes the test. We propose three categories of tests, which differ in the choice of $x_{-}{ }^{3}$ :

1. OVA (One-vs-All): Define $x_{-}=\{y \mid y \in$ $\left.X-x, y \neq x_{+}\right\}$. A model must identify $x$ to be closer to $x_{+}$than all $x_{-}$.

2. SC(Strict Contrastive): Choose $x_{-}$to be the second-closest to $x$ after $x_{+}$under $p$.

3. BC (Broad Contrastive): Choose $x_{-}$to be the furthest from $x$ under property $p$.

\footnotetext{
${ }^{2}$ Several cultures have developed numeration systems (Zhang and Norman, 1995). In this work, we restrict our scope to Arabic and English numeration systems (e.g. Arabic-2, English: two).

${ }^{3} x_{+}$is chosen to be the token closest to $x$ under $p$.
}

\begin{tabular}{|c|c|c|}
\hline Model & \#English & \#Arabic \\
\hline GloVe-6B-*D & $120(0.03 \%)$ & $19409(4.85 \%)$ \\
GloVe-42B-300D & $239(0.01 \%)$ & $108839(5.68 \%)$ \\
GloVe-840B-300D & $532(0.02 \%)$ & $109353(4.98 \%)$ \\
FastText-Wiki & $374(0.04 \%)$ & $25549(2.56 \%)$ \\
FastText-CC & $592(0.03 \%)$ & $59386(2.97 \%)$ \\
SkipGram-BoW & $114(0.06 \%)$ & $2401(1.31 \%)$ \\
SkipGram-Dep & $111(0.06 \%)$ & $2416(1.39 \%)$ \\
\hline GloVe-Num & $1117(0.02 \%)$ & $318109(4.4 \%)$ \\
GloVe-All & $973(0.01 \%)$ & $189598(2.8 \%)$ \\
FastText-Num & $1117(0.02 \%)$ & $317627(4.4 \%)$ \\
FastText-All & $973(0.01 \%)$ & $189366(2.8 \%)$ \\
Word2Vec-Num & $486(0.02 \%)$ & $67908(2.7 \%)$ \\
Word2Vec-All & $434(0.01 \%)$ & $37164(1.2 \%)$ \\
\hline
\end{tabular}

Table 1: Proportion of English and Arabic numerals containing representations in different models. Though embeddings are retrained on the same corpus, preprocessing choices (eg:lowercasing, filtering low frequency words etc.) result in different vocabularies

OVA requires that $x_{+}$must be the closest vector to $x$ in the embedding space. High performance on this test would indicate that the property is captured almost precisely. SC relaxes strictness by only requiring $x_{+}$to be closer than the secondclosest token under property $p$. Finally BC is the least strict of the three. Models can succeed on BC if they manage to capture even an approximate notion of $p$. We use this framework to construct three categories of contrastive tests for both magnitude and numeration. Example tests for magnitude are shown below ${ }^{4}$ :

1. OVA-MAG: $(3,4, x)$, such that $x=\{y \mid y \in$ $X-\{3\}, y \neq 4\}$

2. SC-MAG: $(3,4,5)$

3. BC-MAG: $(3,4,1000000)$

Similarly for numeration,

1. OVA-Num: $(3$, three, $x)$, such that $x=$ $\{y \mid y \in Y, y \neq$ three $\}$

2. SC-NuM: (3, three, four $)$

3. BC-NUM: $(3$, three, billion $)$

\section{Representations}

We evaluate the following embedding methods:

Skipgram (Mikolov et al., 2013a): Feedforward network trained to predict words within a fixed window surrounding the current word, with hidden weights used as embeddings. We evaluate with window sizes in $\{2,5\}$, dependency

\footnotetext{
${ }^{4}$ Note that we consider 2 and 4 equidistant from 3 , so examples like $(3,2,4)$ are removed.
} 


\begin{tabular}{|c|c|c|c|}
\hline Model & OVA-MAG & SC-MAG & BC-MAG \\
\hline Random & 0.04 & 49.82 & 49.34 \\
GloVe-6B-50D & 7.70 & 55.62 & 82.48 \\
GloVe-6B-100D & 10.27 & 57.83 & 82.83 \\
GloVe-6B-200D & 15.88 & 62.21 & 83.94 \\
GloVe-6B-300D & $\mathbf{1 8 . 4 1}$ & $\mathbf{6 2 . 9 2}$ & 83.98 \\
GloVe-42B-300D & 5.18 & 55.58 & 91.86 \\
GloVe-840B-300D & 11.06 & 55.40 & 88.54 \\
\hline FastText-Wiki & 13.94 & 59.96 & 96.15 \\
FastText-CC & 7.83 & 53.89 & 85.40 \\
\hline SkipGram-2 & 7.12 & 55.49 & 95.84 \\
SkipGram-5 & 8.85 & 55.40 & $\mathbf{9 6 . 4 2}$ \\
SkipGram-Dep & 3.32 & 51.99 & 94.60 \\
\hline
\end{tabular}

Table 2: Performance (\% accuracy) of various embedding models on magnitude tests. We also report the performance of a random embedding baseline.

parse-based context (Levy and Goldberg, 2014)

GloVe (Pennington et al., 2014): Embeddings generated by training log-bilinear models to predict global word co-occurrence statistics. We evaluate variants with \#tokens in $\{6 \mathrm{~B}, 42 \mathrm{~B}$, $840 \mathrm{~B}\}$; dimensionality in $\{50,100,200,300\}$

FastText (Bojanowski et al., 2017): Extended Skipgram model representing words as character n-grams to incorporate sub-word information. We evaluate Wikipedia and Common Crawl variants.

\subsection{Retrained Word Vectors}

We retrain all models on GigaWord ${ }^{5}$ and English Wikipedia ${ }^{6}$, under the setting: window size $=5$; dimensionality $=100$. To evaluate whether having more occurrences of numerals in the training data correlates with better representations, we train two variants for each model: one on sentences containing numbers (56M in total; $1.5 \mathrm{~B}$ tokens) (Num), and another on 56M sentences (1.5B tokens) subsampled without constraints (All).

\section{Experiments}

How many numerals have representations? Table 1 shows the proportion of English ${ }^{7}$ and Arabic numerals in each. Overall, numerals make up less than $5 \%$ vocabulary in all models. Despite

\footnotetext{
${ }^{5}$ We use the fourth edition: https : / / catalog. ldc. upenn. edu/LDC2009T13.

${ }^{6}$ We use the May 1, 2019 dump from https:// dumps.wikimedia.org/backup-index.html.

${ }^{7}$ To detect English numerals, we use word2number: https://pypi.org/project/word2number/.
}

this, all variants contain representations for sufficient numerals to allow us to apply our framework.

For off-the-shelf variants, we construct 2260 OVA-MAG, SC-MAG and BC-MAG tests. For numeration, we construct separate tests for each model, as there are few common numerals. Further statistics about number of tests for each model are reported in table 3. For retrained embeddings, we construct 31860 OVA-MAG, SC-MAG and BC-MAG tests, 130 OVA-NUM and SC-NUM tests, and 136 BC-Num tests ${ }^{8}$.

\subsection{Evaluating Off-The-Shelf Embeddings}

Tables 2 and 3 present the performance of offthe-shelf embeddings on magnitude and numeration tests respectively. We use cosine similarity ${ }^{9}$ as the distance metric. High performance on BCMAG indicates that all models capture an approximate notion of magnitude, distinguishing between very large and very small numbers. We speculate this might be because numbers from different magnitude classes often appear in different contexts (See $\$ 5.1$ ). As tests become stricter, model performance drops massively. Models perform nearly $10 \mathrm{x}$ worse on OVA-MAG as compared to BC-MAG. This suggests model are unable to capture magnitude precisely. Across models, SkipGram variants and FastText-Wiki perform best on BC-MAG. However, GloVe outperforms all others on OVA-MAG and SC-MAG. On numeration tests, models fare much worse. With the exception of GloVe models on BC-NUM, no model significantly outperforms a random baseline.

\subsection{Evaluating Retrained Embeddings}

Table 4 presents the performance of retrained embeddings and a random embedding baseline on magnitude and numeration tests. There is no significant difference in performance between Num and All variants, suggesting that seeing more numerals during training does not necessarily result in better representations. Results follow similar trends as off-the-shelf embeddings. All models capture an approximate notion of magnitude (high performance on BC-MAG), but do not capture numeration. Across models, FastText variants fare

\footnotetext{
${ }^{8}$ Since all embeddings are trained on the same corpus and share the same vocabulary, there are enough common English numerals to construct a single set of numeration tests.

${ }^{9}$ We experiment with Euclidean distance, and observe similar results (Appendix A and B).
} 


\begin{tabular}{|c|c|c|c|c|c|c|c|c|c|}
\hline \multirow{2}{*}{ Model } & \multicolumn{3}{|c|}{ OVA-NUM } & \multicolumn{3}{c|}{ SC-NUM } & \multicolumn{3}{c|}{ BC-NUM } \\
\cline { 2 - 10 } & \#Tests & Rand & Emb & \#Tests & Rand & Emb & \#Tests & Rand & Emb \\
\hline GloVe-6B-50D & 117 & 0.00 & 0.85 & 117 & 49.57 & 52.99 & 117 & 50.43 & $\mathbf{7 9 . 4 9}$ \\
GloVe-6B-100D & 117 & 0.00 & 0.85 & 117 & 52.99 & 47.86 & 117 & 57.26 & $\mathbf{8 1 . 2 0}$ \\
GloVe-6B-200D & 117 & 1.71 & 0.85 & 117 & 48.72 & $\mathbf{5 7 . 2 6}$ & 117 & 42.74 & $\mathbf{7 8 . 6 3}$ \\
GloVe-6B-300D & 117 & 1.71 & 0.00 & 117 & 50.43 & $\mathbf{5 8 . 9 7}$ & 117 & 54.70 & $\mathbf{8 8 . 8 9}$ \\
GloVe-42B-300D & 226 & 0.44 & 0.44 & 226 & 52.21 & 51.33 & 226 & 53.98 & 10.18 \\
GloVe-840B-300D & 515 & 0.19 & 0.19 & 515 & 49.90 & 50.68 & 515 & 49.71 & $\mathbf{8 1 . 9 4}$ \\
\hline FastText-Wiki & 360 & 0.28 & 0.28 & 360 & 50.00 & 49.72 & 360 & 56.67 & 41.67 \\
FastText-CC & 572 & 0.00 & $\mathbf{0 . 5 2}$ & 572 & 46.85 & 51.22 & 572 & 41.26 & 44.76 \\
\hline SkipGram-2 & 112 & 0.00 & 0.00 & 112 & 51.79 & 48.21 & 112 & 49.11 & 49.11 \\
SkipGram-5 & 112 & 0.00 & 0.89 & 112 & 52.68 & 51.79 & 112 & 50.89 & 14.29 \\
SkipGram-Dep & 109 & 0.92 & $\mathbf{1 . 8 3}$ & 109 & 53.21 & 48.62 & 109 & 52.29 & 31.19 \\
\hline
\end{tabular}

Table 3: Performance (\% accuracy) of various embedding models on numeration tests. Since we construct a separate set of tests per model, we report the performance of a random embedding model for each set (Rand). Bolded numbers highlight cases where performance is higher than both random embedding and random choice. Note that random choice performance for OVA-NUM is $\frac{1}{\# \text { Tests }}$.

\begin{tabular}{|c|c|c|c|c|c|c|}
\hline \multirow{2}{*}{ Model } & \multicolumn{3}{|c|}{ Magnitude } & \multicolumn{3}{c|}{ Numeration } \\
\cline { 2 - 7 } & OVA-MAG & SC-MAG & BC-MAG & OVA-NUM & SC-NUM & BC-NUM \\
\hline random & 0.00 & 49.62 & 49.71 & $\mathbf{2 . 3 1}$ & 47.69 & 53.68 \\
\hline GloVe-Num & 0.01 & 49.47 & 72.76 & 0.00 & 50.00 & 19.85 \\
GloVe-All & 0.01 & 49.08 & 74.02 & 0.00 & 46.15 & 19.85 \\
\hline FastText-Num & $\mathbf{0 . 0 9}$ & 51.05 & 96.69 & 1.54 & $\mathbf{5 4 . 6 2}$ & 58.09 \\
FastText-All & $\mathbf{0 . 0 9}$ & $\mathbf{5 1 . 1 6}$ & $\mathbf{9 7 . 9 0}$ & 0.00 & 46.92 & $\mathbf{6 1 . 0 3}$ \\
\hline Word2Vec-Num & 0.02 & 50.12 & 93.55 & 0.77 & 44.62 & 33.82 \\
Word2Vec-All & 0.02 & 49.37 & 94.20 & 0.00 & $\mathbf{5 4 . 6 2}$ & 34.56 \\
\hline
\end{tabular}

Table 4: Performance (\% accuracy) of various (retrained) embedding models on magnitude and numeration tests.

best.

\section{Discussion}

\subsection{Performance on Magnitude Tests}

Tables 2 and 4 show that most models do not capture magnitude precisely (low performance on OVA-MAG; SC-MAG), but seem to learn an approximate notion of magnitude (high performance on $\mathrm{BC}-\mathrm{MAG})^{10}$. To examine the difference in contexts that separates numbers of vastly varying magnitudes, we sample 1 million sentences containing numbers from English Wikipedia and GigaWord and compute pointwise mutual information (PMI), defined as

PMI $($ number, class $)=\log \frac{p(\text { number }, \text { class })}{p(\text { number }, \cdot) p(\cdot, \text { class })}$

\footnotetext{
${ }^{10}$ Cognitive studies show that human babies initially start recognizing numbers by approximation and their ability to identify numbers precisely improves over their lifespan (Halberda et al., 2012). (Moyer and Landauer, 1967) were the first to observe that humans took longer to distinguish between closer numbers (eg: 8 and 9) than numbers which were further away in distance (eg: 2 and 9). This finding has since been replicated several times (Dehaene, 2011). In our framework, models find it harder to distinguish between closer numbers (SC-MAG) than distant numbers (BC-MAG)- however the differences here likely arise from different contexts in which numbers of vastly varying magnitudes are used.
}

between the contexts of primitive numbers (numbers 1-10) and large numbers $(>500,>1000$, $>3000,>10000,>100000)$ as shown in Table 5. We consider the word immediately following the number as context, since it appears in the context of the number across embedding methods, regardless of sliding window size. We apply add-100 smoothing to identify contexts with maximum discriminatory power.

We observe in table 5 that terms separating primitives from larger numbers fall into categories such as days in a month, which are less than 31 , or percentages which are $<=100$. In comparison, contexts of larger numbers include terms like 'election', 'census' and 'world'. As we move beyond numbers that are likely to be dates $(>3000)$, we observe terms such as 'ZIP' occurring with ZIP codes in text, 'block' occurring in contexts such as 'house in 9600 block of Washington Boulevard, 'Refugees' which appears in contexts such as 'relocate about 125,000 refugees away from the border'. We observe that different contexts characterize classes of numbers, and speculate that this may allow embeddings to distinguish between numbers that appear consistently in vastly different contexts 


\begin{tabular}{|c|c|c|c|c|c|c|c|c|c|c|}
\hline & Primitives & $\lambda=\mathbf{5 0 0}$ & Primitives & $\lambda=1000$ & | Primitives & $\lambda=\mathbf{3 0 0 0}$ & |Primitives & $\lambda=10000$ & Primitives & $\lambda=100000$ \\
\hline Wiki & $\mid \begin{array}{l}\% \\
\text { July } \\
\text { January } \\
\text { April } \\
\text { September }\end{array}$ & $\begin{array}{l}\text { Summer } \\
\text { Census } \\
\text { Film } \\
\text { World } \\
\text { Election }\end{array}$ & \begin{tabular}{|l}
$\%$ \\
million \\
July \\
January \\
April
\end{tabular} & $\begin{array}{l}\text { Summer } \\
\text { Census } \\
\text { Film } \\
\text { World } \\
\text { Election }\end{array}$ & $\mid \begin{array}{l}\% \\
\text { million } \\
\text { July } \\
\text { January } \\
\text { May }\end{array}$ & $\begin{array}{l}\text { BC } \\
\text { RPM } \\
\text { BCE } \\
\text { Inhabitants } \\
\text { Hollywood }\end{array}$ & $\mid \begin{array}{l}\% \\
\text { million } \\
\text { July } \\
\text { January } \\
\text { May }\end{array}$ & $\begin{array}{l}\text { Exchange } \\
\text { HD } \\
\text { Departs } \\
\text { Delhi } \\
\text { Raxaul }\end{array}$ & $\begin{array}{l}\% \\
\text { million } \\
\text { May } \\
\text { July } \\
\text { January }\end{array}$ & $\begin{array}{l}\text { Elected } \\
\text { Ontario } \\
\text { Owner } \\
\text { Spinneys } \\
\text { Thana }\end{array}$ \\
\hline GW & $\begin{array}{l}\text { percent } \\
\text { p.m } \\
\text { a.m } \\
\text { trillion } \\
\text { billion }\end{array}$ & $\begin{array}{l}\text { index } \\
\text { GMT } \\
\text { World } \\
\text { Olympic } \\
\text { Olympics }\end{array}$ & \begin{tabular}{|l} 
percent \\
million \\
billion \\
p.m \\
years
\end{tabular} & $\begin{array}{l}\text { World } \\
\text { GMT } \\
\text { Olympic } \\
\text { Olympics } \\
\text { season }\end{array}$ & $\begin{array}{l}\text { percent } \\
\text { million } \\
\text { billion } \\
\text { p.m } \\
\text { years }\end{array}$ & $\begin{array}{l}\text { DOWN } \\
\text { Composite } \\
\text { block } \\
\text { LAS } \\
\text { UP }\end{array}$ & $\begin{array}{l}\text { percent } \\
\text { million } \\
\text { billion } \\
\text { points } \\
\text { p.m }\end{array}$ & $\begin{array}{l}\text { novos } \\
\text { ZIP } \\
\text { University } \\
\text { UP } \\
\text { Old }\end{array}$ & $\begin{array}{l}\text { percent } \\
\text { million } \\
\text { billion } \\
\text { points } \\
\text { p.m }\end{array}$ & $\begin{array}{l}\text { novos } \\
\text { Tel } \\
\text { NDI } \\
\text { Refugees } \\
\text { Eritrean }\end{array}$ \\
\hline
\end{tabular}

Table 5: Top 5 nouns by $P M I$ (word, class) for primives and large numbers (numbers $>\lambda$ ), in 1 million sentences drawn from Wikipedia (wiki) and GigaWord (GW) respectively.

leading to good performance on BC-MAG.

\subsection{Recovering magnitude information from nearest neighbours}

Model performance on SC-MAG and BC-MAG indicates whether ordering relationships between a number,its closest, second-closest, and furthest numbers are maintained. However, infinite numbers exist, making it infeasible to construct contrastive tests to check ordering relationships between all triples. To mitigate this, we experiment with a paradigm that performs regression with a number's nearest neighbors to predict its magnitude. If magnitude can be recovered from the structure of the embedding space, this provides evidence that magnitude ordering relations are maintained to some extent. For this experiment, we divide the set of 2260 numbers common across offthe-shelf variants ${ }^{11}$ into training $(80 \%)$ and test (20\%) sets and run a kNN (k-nearest neighbor) regressor model to predict magnitude. R2 scores for are shown in table 6. Most models show reasonably high R2 scores, indicating that some ordering relationships must be maintained, helping embeddings capture approximate notions of magnitude. While this property of current embedding models is interesting, their failure to capture precise magnitude is an important issue. Word embeddings are used for semantic tasks such as natural language inference or reading comprehension, wherein models might need to reason more precisely about numbers.

\section{Conclusion}

Current NLP systems rely heavily on word embeddings. In this work we demonstrate that three

\footnotetext{
${ }^{11}$ We do this to compare results across all models. Retrained variants contain embeddings for all 2260 numbers.
}

\begin{tabular}{|c|c|}
\hline Model & R2 Score \\
\hline GloVe-6B-50D & 0.53 \\
GloVe-6B-100D & 0.75 \\
GloVe-6B-200D & 0.67 \\
GloVe-6B-300D & 0.62 \\
GloVe-42B-300D & 0.44 \\
GloVe-840B-300D & $\mathbf{0 . 8 3}$ \\
\hline FastText-Wiki & 0.71 \\
FastText-CC & 0.56 \\
\hline SkipGram-2 & 0.67 \\
SkipGram-5 & 0.76 \\
SkipGram-Dep & 0.41 \\
\hline GloVe-Num & 0.12 \\
GloVe-All & 0.30 \\
\hline FastText-Num & 0.73 \\
FastText-All & 0.47 \\
\hline Word2Vec-Num & 0.68 \\
Word2Vec-All & 0.65 \\
\hline
\end{tabular}

Table 6: Results of kNN Regression Test for Magnitude

popular embedding models are inadequate at dealing precisely with numbers, in two aspects: magnitude and numeration. We hope this work will promote a more careful treatment of language, and serve a cautionary purpose against using word embeddings in downstream tasks without recognizing their limitations. This work also raises important questions about other categories of word-like tokens that need to be treated like special cases. We hope the community will carefully consider that distributed word representations cannot be relied upon in all scenarios.

\section{Acknowledgements}

This work has partially been supported by the National Science Foundation under Grant No. CNS 13-30596. The authors would like to thank Thomas Manzini, Shruti Rijhwani and Siddharth Dalmia for helpful discussions and reviews while drafting this paper. 


\section{References}

Christian Agrillo, Laura Piffer, Angelo Bisazza, and Brian Butterworth. 2012. Evidence for two numerical systems that are similar in humans and guppies. PloS one, 7(2):e31923.

Piotr Bojanowski, Edouard Grave, Armand Joulin, and Tomas Mikolov. 2017. Enriching word vectors with subword information. Transactions of the Association for Computational Linguistics, 5:135-146.

Jessica F Cantlon and Elizabeth M Brannon. 2006. Shared system for ordering small and large numbers in monkeys and humans. Psychological science, 17(5):401-406.

Ronan Collobert and Jason Weston. 2008. A unified architecture for natural language processing: Deep neural networks with multitask learning. In Proceedings of the 25th international conference on Machine learning, pages 160-167. ACM.

Simone Cutini and Mario Bonato. 2012. Subitizing and visual short-term memory in human and non-human species: a common shared system? Number without language: comparative psychology and the evolution of numerical cognition, 129.

Stanislas Dehaene. 2011. The number sense: How the mind creates mathematics. OUP USA.

Stanislas Dehaene, Ghislaine Dehaene-Lambertz, and Laurent Cohen. 1998. Abstract representations of numbers in the animal and human brain. Trends in neurosciences, 21(8):355-361.

Lisa Feigenson, Stanislas Dehaene, and Elizabeth Spelke. 2004. Core systems of number. Trends in cognitive sciences, 8(7):307-314.

J. Firth. 1957. A synopsis of linguistic theory 19301955. In Studies in Linguistic Analysis. Philological Society, Oxford. Reprinted in Palmer, F. (ed. 1968) Selected Papers of J. R. Firth, Longman, Harlow.

Xavier Glorot, Antoine Bordes, and Yoshua Bengio. 2011. Domain adaptation for large-scale sentiment classification: A deep learning approach. In Proceedings of the 28th international conference on machine learning (ICML-11), pages 513-520.

Hans J Gross. 2011. To bee or not to bee, this is the question the inborn numerical competence of humans and honeybees: The inborn numerical competence of humans and honeybees. Communicative \& integrative biology, 4(5):594-597.

Justin Halberda, Ryan Ly, Jeremy B Wilmer, Daniel Q Naiman, and Laura Germine. 2012. Number sense across the lifespan as revealed by a massive internetbased sample. Proceedings of the National Academy of Sciences, 109(28):11116-11120.

Zellig S Harris. 1954. Distributional structure. Word, 10(2-3):146-162.
Véronique Izard and Stanislas Dehaene. 2008. Calibrating the mental number line. Cognition, 106(3):1221-1247.

Omer Levy and Yoav Goldberg. 2014. Dependencybased word embeddings. In Proceedings of the 52nd Annual Meeting of the Association for Computational Linguistics (Volume 2: Short Papers), pages 302-308, Baltimore, Maryland. Association for Computational Linguistics.

Marie-Catherine Marneffe, Anna N Rafferty, and Christopher D Manning. 2008. Finding contradictions in text. Proceedings of ACL-08: HLT, pages 1039-1047.

Tomas Mikolov, Kai Chen, Greg Corrado, and Jeffrey Dean. 2013a. Efficient estimation of word representations in vector space. arXiv preprint arXiv:1301.3781.

Tomas Mikolov, Ilya Sutskever, Kai Chen, Greg S Corrado, and Jeff Dean. 2013b. Distributed representations of words and phrases and their compositionality. In Advances in neural information processing systems, pages 3111-3119.

Tomas Mikolov, Wen-tau Yih, and Geoffrey Zweig. 2013c. Linguistic regularities in continuous space word representations. In Proceedings of the 2013 Conference of the North American Chapter of the Association for Computational Linguistics: Human Language Technologies, pages 746-751, Atlanta, Georgia. Association for Computational Linguistics.

Jeff Mitchell and Mirella Lapata. 2009. Language models based on semantic composition. In Proceedings of the 2009 Conference on Empirical Methods in Natural Language Processing: Volume 1-Volume 1, pages 430-439. Association for Computational Linguistics.

Robert S Moyer and Thomas K Landauer. 1967. Time required for judgements of numerical inequality. Nature, 215(5109):1519.

Aakanksha Naik, Abhilasha Ravichander, Norman Sadeh, Carolyn Rose, and Graham Neubig. 2018. Stress test evaluation for natural language inference. In Proceedings of the 27th International Conference on Computational Linguistics, pages 2340-2353, Santa Fe, New Mexico, USA. Association for Computational Linguistics.

Jeffrey Pennington, Richard Socher, and Christopher Manning. 2014. Glove: Global vectors for word representation. In Proceedings of the 2014 Conference on Empirical Methods in Natural Language Processing (EMNLP), pages 1532-1543, Doha, Qatar. Association for Computational Linguistics.

David E Rumelhart, Geoffrey E Hinton, Ronald J Williams, et al. Learning representations by backpropagating errors. Cognitive modeling, 5(3):1. 
Bertrand Russell. 2009. Principles of mathematics. Routledge.

Georgios P Spithourakis and Sebastian Riedel. 2018. Numeracy for language models: Evaluating and improving their ability to predict numbers. arXiv preprint arXiv:1805.08154.

Peter D Turney. 2013. Distributional semantics beyond words: Supervised learning of analogy and paraphrase. Transactions of the Association for Computational Linguistics, 1:353-366.

Peter D Turney and Patrick Pantel. 2010. From frequency to meaning: Vector space models of semantics. Journal of artificial intelligence research, 37:141-188

John Whalen, Charles R Gallistel, and Rochel Gelman. 1999. Nonverbal counting in humans: The psychophysics of number representation. Psychological Science, 10(2):130-137.

Jiajie Zhang and Donald A Norman. 1995. A representational analysis of numeration systems. Cognition, 57(3):271-295.

Xunjie Zhu, Tingfeng Li, and Gerard de Melo. 2018. Exploring semantic properties of sentence embeddings. In Proceedings of the 56th Annual Meeting of the Association for Computational Linguistics (Volume 2: Short Papers), pages 632-637, Melbourne, Australia. Association for Computational Linguistics.

\section{A Magnitude Tests with Euclidean Distance}

Table 7 describes the performance of word embedding models on magnitude tests with Euclidean distance.

\begin{tabular}{|c|c|c|c|}
\hline Model & OVA-MAG & SC-MAG & BC-MAG \\
\hline Random & 0.04 & 49.82 & 49.34 \\
GloVe-6B-50D & 7.7 & 54.87 & 79.78 \\
GloVe-6B-100D & 10.27 & 57.12 & 78.5 \\
GloVe-6B-200D & 15.88 & 58.72 & 80.09 \\
GloVe-6B-300D & 18.41 & 60.44 & 79.82 \\
GloVe-42B-300D & 5.18 & 55.27 & 55.09 \\
GloVe-840B-300D & 11.06 & 55.49 & 98.23 \\
\hline SkipGram-2 & 8.85 & 55.35 & 96.37 \\
SkipGram-5 & 7.12 & 55.44 & 95.8 \\
SkipGram-Dep & 3.32 & 51.95 & 94.56 \\
\hline FastText-CC & 7.83 & 54.07 & 91.28 \\
FastText-Wiki & 13.94 & 59.34 & 98.19 \\
\hline
\end{tabular}

Table 7: Performance (\% accuracy) of embedding models on magnitude tests with Euclidean distance

\begin{tabular}{|c|c|c|c|}
\hline \multirow{2}{*}{ Model } & \multicolumn{3}{|c|}{ SC-NUM } \\
\cline { 2 - 4 } & \#Tests & Rand & Emb \\
\hline GloVe-6B-50D & 117 & 49.57 & 52.14 \\
GloVe-6B-100D & 117 & 52.99 & 51.28 \\
GloVe-6B-200D & 117 & 48.72 & 52.65 \\
GloVe-6B-300D & 117 & 50.43 & 56.89 \\
GloVe-42B-300D & 226 & 52.21 & 52.65 \\
GloVe-840B-300D & 515 & 49.90 & 56.89 \\
\hline FastText-Wiki & 360 & 50.00 & 49.72 \\
FastText-CC & 572 & 46.85 & 49.72 \\
SkipGram-2 & 112 & 51.79 & 48.21 \\
\hline SkipGram-5 & 112 & 52.68 & 51.79 \\
SkipGram-Dep & 109 & 53.21 & 48.62 \\
\hline
\end{tabular}

Table 8: Performance (\% accuracy) of embedding models on SC-NUM

\begin{tabular}{|c|c|c|c|}
\hline \multirow{2}{*}{ Model } & \multicolumn{3}{|c|}{ BC-NUM } \\
\cline { 2 - 4 } GloVe-6B-50D & 117 & 50.43 & $\mathbf{9 9 . 1 5}$ \\
\#loVe-6B-100D & 117 & 57.26 & $\mathbf{1 0 0 . 0}$ \\
GloVe-6B-200D & 117 & 42.74 & $\mathbf{2 . 2 1}$ \\
GloVe-6B-300D & 117 & 54.70 & $\mathbf{8 7 . 5 7}$ \\
GloVe-42B-300D & 226 & 53.98 & 2.21 \\
GloVe-840B-300D & 515 & 49.71 & $\mathbf{8 7 . 5 7}$ \\
\hline FastText-Wiki & 360 & 56.67 & 98.89 \\
FastText-CC & 572 & 41.26 & 98.89 \\
SkipGram-2 & 112 & 49.11 & 49.11 \\
\hline SkipGram-5 & 112 & 50.89 & 14.29 \\
SkipGram-Dep & 109 & 52.29 & 31.19 \\
\hline
\end{tabular}

Table 9: Performance (\% accuracy) of embedding models on BC-NUM

\section{B Numeration Tests with Euclidean Distance}

Tables 8 and 9 describe the performance of word embedding models on numeration tests with Euclidean distance. 